Pbilosophia Philosophia Scientiæ

Scientie Travaux d'histoire et de philosophie des sciences

18-2 | 2014

Hugo Dingler et l'épistémologie pragmatiste en

Allemagne

\title{
Pour une lecture continue de Hugo Dingler
}

Norbert Schappacher

\section{(2) OpenEdition \\ Journals}

Electronic version

URL: http://journals.openedition.org/philosophiascientiae/950

DOI: $10.4000 /$ philosophiascientiae.950

ISSN: $1775-4283$

Publisher

Éditions Kimé

\section{Printed version}

Date of publication: 15 June 2014

Number of pages: $105-117$

ISBN: 978-2-84174-672-9

ISSN: 1281-2463

Electronic reference

Norbert Schappacher, « Pour une lecture continue de Hugo Dingler », Philosophia Scientiæ [Online],

18-2 | 2014, Online since 18 June 2015, connection on 02 November 2020. URL : http://

journals.openedition.org/philosophiascientiae/950 ; DOI : https://doi.org/10.4000/

philosophiascientiae.950 


\title{
Pour une lecture continue de Hugo Dingler
}

\author{
Norbert Schappacher \\ Institut de Recherche Mathématique Avancée, \\ Strasbourg (France)
}

Résumé : Au vu, d'une part, de l'intérêt considérable pour certains aspects de la pensée dinglerienne, en particulier pour le fondement des sciences expérimentales, et d'autre part, des textes et des actions de Dingler inspirés par la nazisme de 1933 à 1945, l'article explore la cohérence globale de l'œuvre de Dingler.

Abstract: Given the considerable interest in certain aspects of Hugo Dingler's thought, esp. for foundational questions of the experimental sciences, on the one hand, and the national-socialist slurs in his publications and activities from 1933 through 1945, the article probes the coherence of Dingler's work as a whole.

L'écrivain philosophe Hugo Dingler (1881-1954) occupe une place singulière dans l'histoire de sa discipline en Allemagne. Il est l'unique représentant de cette discipline qui s'est ouvertement et inconditionnellement engagé pour le régime nazi, avec des gestes d'opportunisme et d'antisémitisme outranciers, du début jusqu'à la fin de ce régime. Ses idées philosophiques, plus précisément ses idées pour une philosophie des sciences, ont tout de même continué à nourrir le débat et des travaux dans la communauté académique après 1945, au moins en RFA. Dingler n'est donc pas oublié aujourd'hui, contrairement aux philosophes nazis officiellement soutenus par le régime. Ainsi en fut-il de Hans Heyse - qui fut le chef de la délégation allemande au Congrès international des philosophes à Paris en 1937 (congrès commémorant le tricentenaire du Discours de la méthode et organisé pendant l'Exposition universelle) [Dahms 2013] et qui ruina la célèbre revue Kant-Studien en peu d'années. Également oubliés, Alfred Baeumler et Ernst Krieck, restés célèbres dans les textes de philosophie de l'éducation, qui réussirent à placer chacun son propre compte rendu de la discipline dans le volume offert par la Science Allemande au Führer à l'occasion de ses 50 ans [Rust 1939], faisant ainsi de la philosophie l'unique 
discipline à deux voix dans ce recueil de déférence politique. Baeumler, Heyse et Krieck ne sont étudiés aujourd'hui que par des historiens de la philosophie $\mathrm{du} \mathrm{xx}^{\mathrm{e}}$ siècle, tandis que les idées de Dingler ont été cultivées, principalement par l'École constructiviste d'Erlangen et de Constance, mais aussi par des intellectuels comme Hans Albert et Paul K. Feyerabend qui ont témoigné de la forte impression produite sur eux par la démarche dinglerienne au moins pendant certaines périodes de leur vie ${ }^{1}$. Une impression qui peut aussi nous rappeler que le jeune Rudolf Carnap avait à un moment demandé à Dingler de diriger sa thèse, sans que cela se soit réalisée ${ }^{2}$. Le numéro spécial de Philosophia Scientice dans lequel paraît cet article témoigne d'ailleurs de la considération qu'on porte toujours à la pensée dinglerienne.

Le cas de Hugo Dingler diffère de celui de Martin Heidegger à plusieurs égards. D'abord parce que la lutte politique de ce dernier s'acheva peu de temps après son début fulgurant comme Rektor de l'université de Heidelberg, mais aussi et surtout à cause de la marginalité de Hugo Dingler, qui ne permet pas le même type d'analyse. Cette marginalité marqua toute la carrière de Dingler et se reflète dans sa production. En effet, la position de ausserordentlicher Professor [ " professeur extraordinaire », en quelque sorte 《hors rang »] que Dingler obtint en 1920 à l'université de Munich ne comportait pas de salaire fixe, et s'accompagnait d'une charge de cours de mathématiques élémentaires. Âgé de 51 ans, en 1932, il accéda finalement à une chaire de philosophie, pédagogie et psychologie à la Polytechnique de Darmstadt, chaire jumelée avec la responsabilité d'un institut de formation des maîtres à Mayence, mais il fut mis à la retraite le $1^{\mathrm{er}}$ juillet 1934 à cause de la fermeture de ce Pädagogisches Seminar $^{3}$. Il lui restait certes sa pension, c'est-à-dire $70 \%$ de son salaire, mais ses tentatives pour obtenir un autre poste digne de ses aspirations échouèrent, et du point de vue de l'enseignement, on ne lui confia des charges de cours à l'université de Munich qu'en 1935 et en $1940^{4}$. Les journaux intimes de Dingler montrent qu'il voulait depuis sa jeunesse être professeur d'université, et qu'il s'attendait pendant des années qu'on lui offrît une chaire à Göttingen ou à Berlin. Sa vie personnelle, financièrement serrée avant 1932, était donc aussi

${ }^{1}$ Voir [Feyerabend 1997, 47] ; [Albert 1968, en particulier 37-39], pour une discussion plutôt favorable du principe de la philosophie de Dingler que nous allons suivre par la suite. À noter aussi que le programme tout entier de [Albert 1968], qui traite $\mathrm{du}$ fondement des sciences en parallèle avec celui de l'éthique, etc., suit un projet analogue à celui de Dingler. Voir aussi [Feyerabend \& Albert 2009, vol. I, 132 ; vol. II, $157-158]$.

${ }^{2}$ Sur la proximité entre Carnap et Dingler dans les années vingt, attestée par leur correspondance et par l'article de Carnap Über die Aufgabe der Physik... [Carnap 1923], voir par exemple [Mormann 2000, 17, 41, 55-59].

${ }^{3}$ Nous n'entrons pas ici dans le débat de savoir si cette mise à la retraite fut peutêtre aussi, ou surtout, une sanction politique du professeur que certains hommes au pouvoir auraient regardé comme philosémite. Le fait que Dingler ne fut pas apprécié par Baeumler et Krieck est attesté au moins quelques années plus tard, cf. [Wolters 1992, 318].

${ }^{4}$ Voir [Schorcht 1990, 216-217]. Cf. [Tilitzki 2002, en particulier 200-203]. 
marquée psychologiquement par sa marginalité. Et sur ce plan psychologique, le fait de ne pas avoir tenu plus qu'une semaine au front Ouest en 1914 honte ultime pour l'homme de l'époque - n'a sûrement pas aidé; il le cachait d'ailleurs pudiquement dans les préfaces des livres et autres écrits rédigés pendant la guerre.

Du point de vue professionnel Dingler était donc essentiellement un littéraire «libre », et ceci se ressent aussi dans le style des vingt-cinq livres qu'il publia entre 1907 et $1952^{5}$ et qui se situent entre traités scientifiques et essais généralistes. Même si certains de ses ouvrages demandent du lecteur des connaissances un peu plus poussées en mathématiques ou en physique, l'écriture semble s'adresser d'habitude à un public large, peu spécialisé. Les notes en bas de pages sont plutôt rares et les passages techniques sont généralement entourés par des développements discursifs écrits dans un style décontracté. Les ouvrages de Dingler comportent de nombreux clins d'œil peu précis, par exemple à des auteurs du passé. Loin de toute méthode historiographique, Dingler aime les appels généraux, généreux et souvent métaphoriques au prétendu sens profond d'évolutions culturelles séculaires pour conforter ses points de vue philosophiques. Même si de tels usages argumentatifs d'aperçus historiques se trouvent aussi dans l'œuvre de Heidegger, la facture pédagogique des textes de Dingler permet au lecteur une lecture facile. Ainsi, l'historien s'épargne les soins méthodologiques considérables que Pierre Bourdieu a dû mettre en œuvre pour monter son analyse de la philosophie de Heidegger comme expression, gouvernée par les règles du champ professionnel de la philosophie, des réflexes intellectuels de la révolution conservatrice [Bourdieu 1988].

Je propose donc de suivre essentiellement au fil de ses livres la manière dont l'auteur Hugo Dingler réagissait à son temps et à sa situation. À la fin de la Grande Guerre il publia son étude sur «la culture des $\mathrm{Juifs}^{6}{ }^{6}$, qui propose comme palliatif à la désorientation de ces années «l'or de l'union », exposée la préface du livre, entre la tradition de la Bible hébraïque et celle du rationalisme grec prolongé par le succès de la science des temps modernes et ses reflets philosophiques depuis Kant. Ainsi serait réalisée cette union tant recherchée et désirée entre Verstand et Seele, entre raison et âme, entre science et religion. Cette relative perméabilité de l'œuvre de Dingler pour l'histoire ambiante renforce la pertinence de la lecture de ses textes comme expression des moments historiques vécus par l'auteur. Nous indiquerons par quelques exemples ce qu'on peut obtenir d'une telle approche. Ce qui nous importe dans cette esquisse est de proposer une analyse nouvelle, qui ne tente pas, comme les analyses antérieures, de préserver les pépites dans l'œuvre de Dingler en les séparant des passages inavouables.

${ }^{5}$ Ici je ne compte pas les trois ouvrages mathématiques que Dingler publia en début de carrière, en 1907, 1911 et 1912.

${ }^{6}$ [Dingler 1919]. Ce livre commence d'ailleurs par un tableau imaginaire de la culture mésopotamienne, dont l'unité originelle et mystique entre science et religion se serait ensuite rompue en deux, impulsant la tradition juive d'une part, et la culture grecque de l'autre. 
Plus précisément, si j'ai souhaité rédiger cette petite intervention, c'est d'abord parce que je n'étais pas satisfait de l'étude de Gereon Wolters qui prétend livrer une analyse candide, basée en grande partie sur des journaux intimes, de l'engagement nazi de Dingler à travers une description on ne peut plus accablante de son caractère pitoyable [Wolters 1992]. Ainsi, son comportement entre 1933 et 1945 apparaît comme lié à l'opportunisme chronique d'un petit personnage faible qui alterne entre dépressions et moments d'euphorie nourrie par l'auto-admiration de ses propres écrits. Je ne conteste pas la possibilité d'une telle analyse et je suis conscient de l'ampleur du travail d'archives réalisé par Wolters. Mais je m'intéresse à ce que cette approche psychologique nous cache. Il est curieux de voir, en effet, que Wolters commence son article, dont la suite contient tant de travail d'érudition, par plusieurs affirmations péremptoires prononcées sans la moindre justification ni analyse. Ainsi, Wolters nous rappelle que Dingler avait esquissé « sa conception de la science dès avant la première guerre mondiale »- ce qui est exact - et il continue :

Elle est apolitique et à peine politisable, contrairement aux concepts heideggeriens [Wolters 1992, 258]

et quelques lignes plus loin :

Le motif qui anime [Das treibende Motiv] la philosophie de la science de Dingler, la recherche radicale de la certitude méthodique du savoir, [...], ne peut pas non plus être la base d'une affinité philosophique au national-socialisme. [Wolters 1992, 258259]

Wolters classifie pourtant un ouvrage de Dingler comme proprement nazi : son «Ethique absolue» [Dingler 1935], que Wolters qualifie de «biologiste». Mais il rassure le lecteur que cet ouvrage «n'entretient aucun lien systématique avec sa philosophie de la science » [Wolters 1992, 260]. Et il constate donc finalement une «absence intrinsèque de relation» [innere Beziehungslosigkeit] entre la philosophie dinglerienne de la science et le national-socialisme. Par toutes ces remarques Wolters se place dans la continuité d'autres auteurs qui, confrontés aux taches noires dans l'image de Hugo Dingler, ont essayé d'une manière ou d'une autre, de fragmenter l'œuvre dinglerienne pour isoler des bouts pourris la tranche à laquelle ils tiennent ${ }^{7}$. Autrement dit, Wolters sacrifie le respect de

${ }^{7}$ Le découpage le plus fin de l'œuvre de Dingler que j'ai vu se trouve dans l'introduction de Ulrich Weiß à l'édition des œuvres complètes de Dingler sur cédérom [Dingler 2004]. Voir sa Selektive Verlaufsskizze de l'œuvre dinglerienne [Dingler 2004, 32-33]. Là aussi, l'exercice sert à marginaliser l'importance des dérapages nazis de Dingler. Ainsi on lit par exemple : « Dans le tableau de l'œuvre complète le "Dingler politique" occupe un secteur très étroit » [Dingler 2004, 52]. Weiß, professeur de théorie politique à l'université de l'armée allemande, n'en reste pourtant pas là. Il propose ensuite de caractériser les écrits politiques de Dingler comme ceux où la pensée de l'auteur [Dingler 2004, 54] « perd sa mesure méthodique et auto-critique et la sûreté systématique qui va avec »[... sein methodisch-sebstkritisches Maß und die damit verbundene systemimmanente Sicherheit verliert.] On nous excusera si nous ne suivons pas plus loin un tel exercice de classification qui ne sert qu'à évacuer ce dont on savait d'avance vouloir éliminer. 
la personne de Dingler afin de sauver sa philosophie de la science. La catégorie de l'opportunisme qu'il emploie, et pour laquelle il regrette de ne pas avoir trouvé de référence méthodologique [Wolters 1992, 254, note 12], focalise bien l'attention sur les intérêts strictement personnels de Dingler, et n'invite donc pas à revenir sur les affirmations initiales de Wolters que nous venons de citer. $\mathrm{Si}$, au lieu de parler d'opportunisme, on tient compte du fait que - pour citer la célèbre formule de Mitchell Ash [Ash 2002] - les domaines politique et scientifique fonctionnent souvent comme ressources l'un pour l'autre, on sera d'emblée sceptique par rapport à des arguments qui prétendent déterminer in abstracto l'incompatibilité de certains philosophèmes avec une idéologie ou politique donnée.

Ces affirmations de Wolters ne passent pas l'épreuve d'une lecture directe des ouvrages de Dingler. Par exemple, le philosophème de base de la refondation méthodique de la science selon Dingler, et qui revient dans la plupart de ses écrits sous une forme ou une autre, consiste à stopper la régression infinie qu'on entame quand on demande des justifications des premiers principes ou axiomes d'une science, par un geste capable de s'auto-justifier. Ce dernier, selon Dingler, ne peut être qu'une résolution de notre « volonté active », exercée conformément au principe de Mach de la plus grande simplicité possible. Mais fonder ainsi toutes les sciences, ou toute la science, sur la volonté de l'homme est évidemment un lemme qui est facilement «politisable »-même si l'écrivain Dingler lui-même avait tendance à réserver le mot «politique » au champ d'action propre des politiciens professionnels, des partis, etc., et n'aurait donc pas employé ce terme pour les premières instances que je vise ici.

Dans Die Kultur der Juden [Dingler 1919] l'union entre la science et la religion est construite à partir de l'analyse dinglerienne des principes et lois scientifiques comme résultant d'un « choix, certes pas arbitraire, mais tout de même libre »[Dingler 1919, 38]. La conséquence pour la vie de chacun en est que

je dois me soumettre dans mes actions aux lois de la réalité qui ont été trouvées, car ces lois s'appliquent à tous les égards à moi et à mes expressions. Il n'existe pas d'opposition contre elles. [Dingler 1919, 43]

Puis, après le développement parallèle de la notion de loi de la Bible, Dingler conclut qu'un même acte volontariste nous fait - des profondeurs de nous-mêmes - accepter aussi la loi morale (« Dieu») tout comme il nous guide vers les lois de la physique.

Il en résulte comme conséquence importante pour nos actions : Si je veux agir dans le sens des buts les plus profonds de ma vie, donc si je veux agir dans le sens de Dieu, je le ferai d'autant plus que j'agis à partir de mon for intérieur. [Dingler 1919, 77] Ainsi,

[nous] sommes tous assujettis à une grande loi de l'existence, nous sommes lourdement punis pour toute déviation, mais - nous ne 
connaissons pas cette loi tout à fait.... Tout ce que nous savons est que son sens doit être la conservation de la vie et de l'humanité jusqu'au plus lointain possible, ainsi que la victoire triomphale sur toutes les résistances intérieures ou extérieures à elle. [Dingler 1919, 89]

Dingler envisage explicitement [Dingler 1919, 90] la possibilité que le Décalogue puisse aussi se révéler un jour injustifié au vu de la «loi fondamentale » [Grundgesetz] que nous sentons indistinctement en nous. Il imagine par exemple une constellation éventuelle où même l'interdiction de l'inceste, malgré son fondement biologique apparent, pourrait se révéler contradictoire avec la loi fondamentale.

Ce parallélisme explicite entre la philosophie des sciences et le fondement de l'éthique est repris et encore élargi chez Dingler environ dix ans plus tard dans des livres comme Metaphysik als Wissenschaft vom Letzten [Dingler 1929], Das System [Dingler 1930], Der Zusammenbruch der Wissenschaft und der Primat der Philosophie [Dingler 1931], et Der Glaube an die Weltmaschine und seine Überwindung [Dingler 1932]. Le noyau commun volontariste des sphères scientifique et irrationnelle lui permet alors d'assigner à la science sa place et son rôle délimités dans la condition humaine, tout en l'intégrant dans un tableau cohérent. Ainsi, la section 21 du livre [Dingler 1930] est intitulée : Das System eingebettet in das Leben [Le système intégré dans la vie]. Nous y lisons notamment que le système scientifique tout entier

n'est pas quelque chose qui nous porte nous-mêmes ou la vie, mais que d'abord il n'y a que la vie et rien que la vie, et qu'à partir d'elle se constitue comme un outil pratique, comme le moyen le plus sûr et couronné de succès dans la lutte pour la vie de l'humanité, le Fsystème [scientifique], venant lui-même de la vie, à jamais intégré dans la vie et formant une (petite) part d'elle. [Dingler 1930, 117]

Et c'est précisément cette analyse qui amène Dingler, dans la section suivante 22 du même livre, intitulée : Die Wendung von der passiven zur aktiven Einstellung [Le revirement de l'attitude passive vers l'attitude active], à fustiger toute recherche sur les fondements des sciences qui procède par voie passive, purement théorique ou contemplative :

Dans les sciences individuelles [exactes] l'activité manuelle a toujours existé de manière éminente, mais au fond on ne l'a pas considérée comme pleinement acceptable du point de vue scientifique, elle a dû se contenter comme ancilla theoriae dans l'antichambre d'un rôle comme subordonné, tandis que la théorie dans la salle du trône présentait ses cabrioles intellectualistes et se croyait le sens du monde. [Dingler 1930, 123]

On n'est donc pas surpris de trouver dans l'introduction du livre sur la métaphysique [Dingler 1929] la note infrapaginale suivante : 
À quel point des dirigeants [Führer] politiques - et au fond ceci va de soi - sont guidés dans leurs actions par leur ultime philosophie, et comment ces opinions affectent ensuite ceux qu'ils guident (die Geführten), on peut le voir - pour ne citer qu'un exemple - dans un traité philosophique de V.I. Lénine, Matérialisme et Empiriocriticisme, où il discute de manière assez pertinente des fondements épistémologiques et avance quelques arguments assez frappants contre le sensualisme, mais où il réinterprète, hélas, le réalisme qu'il défend comme un grossier matérialisme métaphysique. [Dingler 1929]; [Dingler 2004, 1483]

En lisant le livre de 1931, nous rencontrons finalement chez Dingler un principe qui - dans une sorte de fondement de toute philosophie et science par un ultime cercle justificateur - représente d'une part l'ultime motif de toutes les «actions pures», dont notamment celles à la base de toute science, et qui est à son tour expliqué par la «synthèse pure» de toute l'analyse de la condition humaine [Dingler 1931, 377]. C'est ce que Dingler nomme l'Artdauererhaltungstrieb [instinct de conservation durable de l'espèce]. Conscient de ce que peut avoir de choquante l'idée qu'un rôle philosophique tellement éminent soit donné à un instinct, Dingler rassure ses lecteurs [Dingler 1931, 374-375] sur l'analogie de ce principe avec « les tautologies dont la substruction causale a constamment besoin », notamment dans le fondement de la physique.

À la fin des années vingt, Hugo Dingler était donc objectivement fort bien positionné pour pouvoir offrir le moment venu ses idées à une idéologie biologiste anti-intellectuelle prête à accepter la volonté et l'action comme valeurs de base. Dans la constellation concrète du national-socialisme, il fallait juste encore recadrer la source primordiale du but suprême de l'éthique, l'Artdauererhaltungstrieb, sous une forme germanocentrique, comme la Dauererhaltung der Volksgemeinschaft als lebendiger Teil der Menschheit [conservation durable de la communauté du peuple comme part vivante de l'humanité] [Dingler 1934, 619], étape que Dingler franchit apparemment peu de mois après l'arrivée de Hitler au pouvoir.

La continuité et la cohérence interne de l'évolution de la pensée dinglerienne jusqu'aux positions nazies, que nous venons d'indiquer rapidement, ont aussi été soulignées par Claudia Schorcht dans son étude de la philosophie dans les universités bavaroises entre 1933 et 1945, voir [Schorcht 1990, 318328]. Elle était pourtant encore obligée de réfuter d'abord l'idée avancée en 1965 par Silagi - en suivant une trame de défense de Dingler lui-même - selon laquelle tous les passages nazis dans l'œuvre de Dingler seraient en fait ironiques, et auraient été compris par ses lecteurs de l'époque comme caricatures de l'idéologie nazie...

Dans l'article de Wolters cité plus haut, le livre de Schorcht est mentionné comme «précieux et riche en matériel » [Wolters 1992, 261, note9], mais l'étude de Schorcht n'a pas empêché Wolters par exemple d'étiqueter l'« éthique absolue » de Dingler (1935) comme « éthique biologiste», comme si ceci différenciait cette œuvre nazie de la production dinglerienne d'avant 
1933 ou d'après 1945. Or, nous avons déjà vu l'exemple de l'Artdauererhaltung - ce terme apparaît dans les livres de Dingler en 1929. Et plus généralement les sciences biologiques sont tout à fait présentes dans les livres de Dingler bien avant. Dans [Dingler 1919] il insiste ainsi sur leur analogie de principe avec des sciences comme la physique ou la géométrie, malgré la plus grande complexité d'une souris par rapport à un morceau de sel [Dingler 1919, par exemple, 41]. D'un autre côté, le philosophème abstrait du fondement auto-justifiant sans regression infinie figure bel et bien d'emblée dans l'Absolute Ethik [Dingler 1935] comme §2. Der Ausgangspunkt, «le point de départ» de ce traité. Dingler a pourtant ajouté certains nouveaux éléments à son argumentaire et à ses références pendant les années 1930, par exemple la référence à Francis Galton et à ses contributions aux statistiques de la corrélation dans un contexte d'eugénisme. Et ce n'est apparemment que dans les années trente et à travers son contact avec le SS-Ahnenerbe (l'Office de la SS chargé des questions d'hérédité) que Dingler commença à s'intéresser aux travaux de Hermann Wirth sur la paléoépigraphie nordique...

Nous avons suivi une trame importante de continuité dans l'œuvre publiée de Hugo Dingler, qui inclut ses textes d'inspiration nazie. La continuité ne masque pourtant pas tout. Jetons donc, pour finir, un coup d'œil à un texte qui n'était pas destiné à être publié : le mémorandum (Denkschrift) par lequel Hugo Dingler essayait de promouvoir en 1933 une nouvelle politique scientifique, concrètement la réorganisation de la formation en mathématiques et physique des futurs professeurs du secondaire. Leur culture en épistémologie, logique et philosophie (au programme de la troisième année d'études préconisé par Dingler) ne devait surtout pas être confié à des enseignants venant « du cercle d'Einstein avec son orientation bolchévique (le soi-disant cercle de Vienne, la Gesellschaft für wissenschaftliche Philosophie à Berlin et le cercle de la revue Erkenntnis)» [p. 25-26]. Le document est intitulé « Développements aberrants dans le domaine et dans la pédagogie des mathématiques et des sciences exactes au cours du dernier demi-siècle ${ }^{8} »$ et présente le sombre tableau des sciences exactes qui seraient tombées sous l'influence des Juifs depuis que l'accès aux postes académiques, à « la science rémunérée par l'État » [p. 2], leur a été ouvert vers le milieu du XIX ${ }^{\mathrm{e}}$ siècle. Incapables de concevoir des vraies contributions profondes à la science, mais dotés de davantage d' $\mathrm{d}^{\text {habileté, de }}$

\footnotetext{
${ }^{8}$ Abwegige Entwicklungen im Gebiete und in der Pädagogik der Mathematik und der exakten Naturwissenschaften im letzten halben Jahrhundert von Prof. Dr. Hugo Dingler, Darmstadt. Le document est présenté comme une synthèse de deux textes plus complets datant respectivement d'avril et de novembre 1933. Le tout date donc d'une époque où Dingler avait encore son poste à Darmstadt. J'ai consulté la retranscription au Geheimes Staatsarchiv à Berlin-Dahlem : GStA PK, I. HA Rep. 76 Kultusministerium, Va Sekt.1 Tit. VII Nr.14. Le texte de Dingler y commence feuille 48. Schorcht cite la version du document dans le Hauptstaatsarchiv à Munich [Schorcht 1990, 218]. Le physicien Philipp Lenard l'avait envoyé le 12/11/1933 au Staatsminister Schemm à Munich. Il a ensuite été jugé suffisamment intéressant pour être communiqué le 21/12/1933 au ministère de l'Intérieur du Reich à Berlin. L'un des fonctionnaires de ce ministère demanda d'ailleurs une copie pour son usage personnel dans une note au crayon sur la feuille $46 \mathrm{du}$ document à Berlin.
} 
vitesse et puissance de la mémoire », au prix d'un «manque de profondeur » [p. 5], les scientifiques juifs étaient conduits à s'imposer à force d'une « production casuistique»surabondante, et en contrôlant de plus en plus les positions du pouvoir académique et la presse.

Le mémorandum de Dingler n'est pas une découverte : David Rowe l'a déjà utilisé comme point d'entrée de son étude très connue [Rowe 1986]. En fait, ce qui nous paraît le plus absurde aujourd'hui dans le texte de Dingler - Rowe l'appelle «patently ludicrous »- est que le maître penseur de la conspiration juive dans le domaine des mathématiques, selon Dingler, est Felix Klein, dont Dingler dit que «l'un au moins de ses parents étaient juifs » [p. 3]. D'où la correction dans le Göttinger Tageblatt que Rowe cite, et l'article [Manger 1934] dans le Jahresbericht der Deutschen Mathematiker-Vereinigung qui démontre - arbre généalogique à l'appui - que Klein était « à même de satisfaire aux exigences raciales les plus élevées ». Toutes ces rectifications choquées de l'erreur de tir de Dingler ne font évidemment que confirmer le fondement antisémite de l'affaire.

Nous ne chercherons pas à savoir si Dingler était antisémite «au fond» ou de manière conjoncturelle, question parfois discutée dans la littérature sur Dingler - voir par exemple [Wolters 1992, 272-275], qui cite des réactions de Dingler par rapport à son professeur Alfred Pringsheim et lors de rencontres avec des groupes de mathématiciens. Toujours est-il qu'il était prêt en 1933 à épingler Felix Klein comme instigateur d'une évolution néfaste pour les mathématiques, centrée à Göttingen. On peut donc se demander si cette attitude de Dingler se reflète dans l'image de Felix Klein que Dingler véhicule dans ses publications, sachant qu'il n'y aura plus de remarques antisémites contre Klein après 1934. En parcourant le cédérom [Dingler 2004], nous avons relevé 37 passages où le nom de Felix Klein est commenté par Dingler. Ces passages varient selon l'aspect de l'œuvre de Felix Klein qui est mis en valeur, et un seul aspect seulement - sans doute le plus important pour l'image de Klein chez Dingler - nous permet de constater une évolution au fil des années : ce sont les endroits où Klein est présenté comme

le premier qui a reconnu l'essence de la différence entre mathématiques pures et appliquées. [...] Dans son évaluation du prix Beneke, il pose le problème des mathématiques appliquées, autrement dit, la question de la précision ${ }^{9}$.

En 1913-1914, Klein se trouve le plus souvent cité dans ce contexte en même temps que Ernst Mach et Aurel Voss. Ensuite, Hilbert est souvent mentionné en même temps, notamment quand Dingler cite à la fois Klein, pour la sépa-

\footnotetext{
${ }^{9}$ Voir [Dingler 2004, 265-266 (1911)]. Le texte de Klein auquel Dingler fait allusion ici se trouve dans Mathematische Annalen 55 (1902), 143-148. Voici d'autres endroits représentatifs de ce point de vue : [Dingler 2004, 3543 (1911)], le discours de Dingler à Bologna en 1911, [Dingler 2004, 3568 (1914)], [Dingler 2004, 3574 (1916)] et [Dingler 2004, 642, 764 (1919)].
} 
ration entre mathématiques pures et appliquées, et les recherches de Hilbert sur l'axiomatisation ${ }^{10}$.

À cette reconnaissance du rôle précurseur se mêle une certaine réserve, certainement dès 1923. Voir par exemple :

Ainsi F. Klein posait la question, de savoir s'il était mieux de décrire les processus naturels de manière atomiste ou par des considérations de continuité, et il a traité la question de la représentation des courbes de manière particulièrement intuitive. Mais un sujet de prix qui allait dans cette direction ne suscitait aucune réponse. La question de l'application ne peut pas être traitée de façon purement mathématique, mais seulement dans la méthodologie. [Dingler 2004, 1042]

Dans Das Experiment [Dingler 1928], Klein est d'abord cité de la même manière qu'auparavant [Dingler 1928, 40, 47] dans les passages rétrospectifs, mais plus loin [Dingler 1928, 93] Dingler constate chez Klein «l'absence totale de contact avec la pratique » parce qu'il ne peut imaginer la fabrication de règles pour la géométrie pratique que «par des lignes de mire ». Ce point revient en 1932, voir [Dingler 2004, 2336], et en 1951 devient un motif pour ridiculiser «le mathématicien génial Felix Klein » :

Qu'on essaie de faire cela! Or il y avait beaucoup de personnes dans tous les États civilisés qui savaient pertinemment bien le secret. [Dingler 2004, 3104]

Dans le courant des années 1920 le respect pour le rôle pionnier de Klein commence donc à être remplacé par le propre point de vue dinglerien. Ceci n'est peut-être qu'une évolution naturelle. Mais elle a lieu quand l'influence de Felix Klein diminuait - il meurt en 1925 à l'âge de 76 ans - et quand les commentaires ne sont plus tenus par l'esprit d'allégeance de l'ancien étudiant de Göttingen qui espérait jadis être élu au même endroit. Ainsi il s'exprime plus librement.

Mais la «liberté » du mémorandum (Denkschrift) est d'un autre registre. Le projet kleinéen de l'Enzyklopädie der mathematischen Wissenschaften y apparaît comme faisant partie intégrante de la conspiration juive pour contrôler le monde mathématique. - En 1915 Dingler l'avait encore accueillie comme

une œuvre multi-volumes que seule la force et la Gründlichkeit allemande est capable d'accomplir. [Dingler 2004, 4282]

Et en 1931 Dingler se moquait encore - au nom de sa propre analyse de l'histoire récente de la science - de l'idée que la race juive aurait précipité, par sa nature, le déclin actuel des sciences [Dingler 1931, 122].

Rien dans les publications de Dingler ne semble donc préparer les invectives du mémorandum contre Felix Klein.

${ }^{10}$ Voir par exemple [Dingler 2004, 3605 (1920)], [Dingler 2004, 944 (1921), 1042, 1089 (1923)]. 
Le 9 janvier 1935, Hedwig Pringsheim - la femme du mathématicien Alfred Pringsheim, qui avait été le garant de l'habilitation de Hugo Dingler en 1912, et belle-mère de Thomas Mann - répondit « de manière très aimable et amicale » à un billet de Maria Dingler, née Stach von Goltzheim, qui avait demandé de pouvoir faire une visite : qu'une telle visite n'était désormais plus possible, au vu de la position par rapport aux Juifs que son mari avait exprimé dans son mémorandum. Les Pringsheim connaissaient Maria Dingler depuis plus de trente ans (quand elle était encore mariée avec Theodor Lessing, philosophe et écrivain politique juif, d'ailleurs assassiné le 31 août 1933 à Marienbad). Le 12 janvier 1935 les deux femmes se rencontrèrent par hasard [?] dans la rue, se promenèrent un peu ensemble, et Maria Dingler expliqua à Hedwig Pringsheim que la raison du mémorandum avait été que des Juifs avaient détruit par des calomnies la possibilité d'une mutation avantageuse pour Hugo Dingler. Maria Dingler souligna que la source de son histoire était «si indubitablement sûre qu'aucun doute [n'était] possible ». Sur quoi Hedwig Pringsheim écrivit à sa fille, Katia Mann ${ }^{11}$ :

Moi je trouve que toutes les sources indubitables sont troubles $[\ldots]$.

\section{Remerciements}

J'ai profité de nombreuses remarques lors de conférences sur Hugo Dingler que j'ai faites devant l' "Académie Helmholtz » et à Francfort. J'en remercie tout particulièrement Moritz Epple, Oliver Schlaudt et Florian Schmaltz. Je remercie Catherine Goldstein d'avoir revu et amélioré une première version de ce texte, et l'équipe de Philosophia Scientice pour sa compétence et son efficacité.

\section{Bibliographie}

Albert, Hans [1968], Traktat über kritische Vernunft, Tübingen : Mohr (Siebeck), 5 éd., 1991.

Ash, Mitchell [2002], Wissenschaft und Politik als Ressourcen füreinander, dans Wissenschaften und Wissenschaftspolitik - Bestandsaufnahmen $z u$ Formationen, Brüchen und Kontinuitäten im Deutschland des 20. Jahrhunderts, édité par R. vom Bruch \& B. Kaderas, Stuttgart : Steiner, $32-51$.

Bourdieu, Pierre [1988], L'Ontologie politique de Heidegger, Paris : Éditions de Minuit.

${ }^{11}$ Voir les lettres et commentaires dans [Pringsheim 2013, 196-202; 596-604] 
Carnap, Rudolf [1923], Über die Aufgabe der Physik und die Anwendung des Grundsatzes der Einfachstheit, Kant-Studien, 28, 90-107.

Dahms, Hans-Joachim [2013], Leibniz auf den internationalen Philosophiekongressen in Prag und Paris (1934 und 1937), dans „Leibniz“ in der Zeit des Nationalsozialismus, édité par W. Li \& H. Rudolph, Stuttgart : Steiner, Studia Leibnitiana, t. Sonderhefte 42, 185-202.

Dingler, Hugo [1919], Die Kultur der Juden. Eine Versöhnung zwischen Religion und Wissenschaft, Leipzig : Verlag Der Neue Geist.

— [1928], Das Experiment, sein Wesen und seine Geschichte, Munich : E. Reinhardt.

— [1929], Metaphysik als Wissenschaft vom Letzten, Munich : E. Reinhardt.

— [1930], Das System. Das philosophisch-rationale Grundproblem und die exakte Methode der Philosophie, Munich : E. Reinhardt.

- [1931], Der Zusammenbruch der Wissenschaft und der Primat der Philosophie, Munich : E. Reinhardt, 2. verbesserte und durch einen Anhang vermehrte Auflage.

— [1932], Der Glaube an die Weltmaschine und seine Überwindung, Stuttgart : F. Enke.

— [1934], Zur Philosophie des Dritten Reiches, Zeitschrift für Deutschkunde (année 48 de Zeitschrift für den deutschen Unterricht), 48, 609-622.

— [1935], Das Handeln im Sinne des höchsten Zieles (Absolute Ethik), Munich : E. Reinhardt.

[2004], Gesammelte Werke auf CD-Rom, Literatur im Kontext auf CD$R O M$, t. 18, Berlin : InfoSoftWare, herausgegeben von Ulrich Weiß, unter Mitarbeit von Silke Jeltsch und Thomas Mohrs, Copyright Karsten Worm.

Feyerabend, Paul K. [1997], Zeitverschwendung, Francfort/Main : Suhrkamp.

Feyerabend, Paul K. \& Albert, Hans [2009], Briefwechsel, VienneKlagenfurt : Kitab-Verlag, Hrsg. W. Baum, M. Mühlmann. 2 vol.

Manger, E. [1934], Felix Klein im Semi-Kürschner!, Jahresbericht der Deutschen Mathematiker-Vereinigung, 44, 4-11.

Mormann, Thomas [2000], Rudolf Carnap, Munich : Beck.

Pringsheim, Hedwig [2013], Mein Nachrichtendienst. Briefe an Katia Mann 1933-1941, t. 1, Göttingen : Wallstein Verlag, Hrsg. von Dirk Heißerer. 
Rowe, David [1986], "Jewish mathematics" at Göttingen in the Era of Felix Klein, Isis, 77, 422-449.

Rust, Bernhard (éd.) [1939], Deutsche Wissenschaft - Arbeit und Aufgabe. Dem Führer und Reichskanzler legt die deutsche Wissenschaft zu seinem 50. Geburtstag Rechenschaft ab, über ihre Arbeit im Rahmen der ihr gestellten Aufgabe, Leipzig : Hirzel.

Schorcht, Claudia [1990], Philosophie an den bayerischen Universitäten 1933-1945, Erlangen : Harald Fischer Verlag.

Tilitzki, Christian [2002], Die deutsche Unversitätsphilosophie in der Weimarer Republik und im Dritten Reich, t. Teil I., Berlin : Akademie Verlag.

Wolters, Gereon [1992], Opportunismus als Naturanlage. Hugo Dingler und das «Dritte Reich», dans Entwicklungen der methodischen Philosophie, édité par P. Janich, Francfort/Main : Suhrkamp, 257-327. 